\title{
Complete solution for unambiguous discrimination of three pure states with real inner products
}

\author{
H. Sugimoto, T. Hashimoto, M. Horibe, and A. Hayashi \\ Department of Applied Physics, University of Fukui, Fukui 910-850\%, Japan
}

\begin{abstract}
Complete solutions are given in a closed analytic form for unambiguous discrimination of three general pure states with real mutual inner products. For this purpose, we first establish some general results on unambiguous discrimination of $n$ linearly independent pure states. The uniqueness of solution is proved. The condition under which the problem is reduced to an $(n-1)$-state problem is clarified. After giving the solution for three pure states with real mutual inner products, we examine some difficulties in extending our method to the case of complex inner products. There is a class of set of three pure states with complex inner products for which we obtain an analytical solution.
\end{abstract}

\section{INTRODUCTION}

In quantum mechanics, there is no way to distinguish nonorthogonal states perfectly. This is because quantum measurement is statistical in nature, and it generally destroys the state of the system to be measured. Considerable works have been done to find optimal ways to distinguish quantum states in various situations [1 3$]$. These studies are stimulated by needs of quantum communication and quantum cryptography where the discrimination of quantum states is one of the key issues.

In the quantum state discrimination problem, two settings have been commonly investigated. In minimumerror discrimination [1], the discrimination success probability is maximized without any constraint on the probability of erroneous results. In unambiguous discrimination, however, the success probability is maximized under the condition that measurement should not produce erroneous results. This is possible by allowing an inconclusive result: "I don't know." Ivanovic [4], Dieks [5], and Peres [6] found the optimal solution for unambiguous discrimination of two pure states with equal occurrence probabilities. Later the case of general occurrence probabilities was solved in Ref. 7]. Unambiguous discrimination was experimentally demonstrated in Ref. [8]. A scheme that interpolates the two discrimination settings has also been proposed, and its solution for two general pure states was given in Refs. [9, 10].

For unambiguous discrimination among more than two pure states, it is not easy to obtain analytical solutions, though a great deal of works on general theories have been reported: For example see Refs. [11 19]. The same thing is true for unambiguous discrimination for mixed states [20 27].

The case of three pure states has also been considered as an application by some articles dealing with general theories. Even for three pure states, to the best of our knowledge, complete analytical results are known only in limited special cases: (i) The case of symmetric states with equal occurrence probabilities [12], in which the states are generated by a single unitary operator; (ii) the case in which two of the three real mutual inner products are equal and equal occurrence probabilities are assumed [14]; and (iii) the case of three pure states with general occurrence probabilities and one of the mutual inner products being 0 [19].

The purpose of this article is to give complete analytical solutions for unambiguous discrimination of three pure states with real mutual inner products and general occurrence probabilities. In unambiguous discrimination, the optimal strategy may produce a vanishing probability of identifying the input state with some of the states. This issue will be thoroughly analyzed.

In Sec. III, we first establish some general results on unambiguous discrimination of $n$ pure states. We formulate the problem as semidefinite programming. It turns out very useful to consider a problem in which some constraints in the problem are relaxed. This relaxed problem is used to clarify the conditions under which some identification probabilities vanish, and the problem is reduced to a certain $(n-1)$-state problem. Then, complete analytical solutions of three pure states with real mutual inner products are presented in Sec. III In Sec. IV] we discuss some difficulties in extending our method to the case of general complex inner products. In the appendix, we show that solutions of unambiguous discrimination for linearly independent $n$ pure states are generally unique.

\section{GENERAL CONSIDERATION}

\section{A. Formulation of problem}

Suppose that a state is drawn from a set of known $n$ pure states $\left|\phi_{i}\right\rangle$ with probabilities $\eta_{i}$. We assume that the $n$ states are linearly independent and the occurrence probabilities $\eta_{i}$ are nonzero. The task is to unambiguously identify the given state with one in the set of $n$ states: An erroneous identification is not allowed, but an inconclusive result ("I don't know") is possible.

Measurement is described by a positive operatorvalued measure (POVM), and it consists of $n+1$ elements $\left\{E_{1}, E_{2}, \ldots, E_{n}, E_{\text {? }}\right\}$, each element being a positive semidefinite operator on the $n$-dimensional space $V$ spanned by $\left|\phi_{i}\right\rangle$. When the outcome is $i(i=1, \ldots, n)$, the given input state is identified with the state $\left|\phi_{i}\right\rangle$. The element $E_{\text {? }}$ corresponds to the inconclusive result. 
The POVM satisfies the following completeness relation:

$$
E_{?}+\sum_{i=1}^{n} E_{i}=\mathbf{1}_{V}
$$

The no-error conditions are written as

$$
\left\langle\phi_{j}\left|E_{i}\right| \phi_{j}\right\rangle=0 \quad(i \neq j),
$$

which require that the support of the positive semidefinite operator $E_{i}$ be the one-dimensional subspace that is orthogonally complement to the $(n-1)$-dimensional subspace spanned by the states $\left\{\left|\phi_{j}\right\rangle\right\}_{j(\neq i)}$. Therefore, $E_{i}$ should take the following form [11]:

$$
E_{i}=x_{i}\left|\tilde{\phi}_{i}\right\rangle\left\langle\tilde{\phi}_{i}\right|
$$

where $x_{i}$ is a non-negative constant to be determined, and the state $\left|\tilde{\phi}_{i}\right\rangle$ is orthogonal to any state $\left|\phi_{j}\right\rangle$ for $j \neq i$. For convenience we normalize the states $\left|\tilde{\phi}_{i}\right\rangle$ such that

$$
\left\langle\tilde{\phi}_{i} \mid \phi_{j}\right\rangle=\delta_{i j}
$$

Since the states $\left|\phi_{i}\right\rangle$ are linearly independent, those states $\left|\tilde{\phi}_{i}\right\rangle$ are uniquely given by

$$
\left|\tilde{\phi}_{i}\right\rangle=\sum_{j=1}^{n}\left(N^{-1}\right)_{j i}\left|\phi_{j}\right\rangle
$$

where

$$
N_{i j} \equiv\left\langle\phi_{i} \mid \phi_{j}\right\rangle
$$

is the Gram matrix of the states to be discriminated.

The coefficient $x_{i}$ represents the conditional probability of successfully identifying an input state given that the input state is $\left|\phi_{i}\right\rangle$. In terms of $x_{i}$, the discrimination success probability is given by

$$
p=\sum_{i=1}^{n} \eta_{i}\left\langle\phi_{i}\left|E_{i}\right| \phi_{i}\right\rangle=\sum_{i=1}^{n} \eta_{i} x_{i} .
$$

Now let us examine what condition the positivity of $E_{\text {? }}$ further imposes on the coefficients $x_{i}$. By the completeness relation (11), the positivity of $E_{\text {? }}$ is expressed as

$$
\mathbf{1}_{V}-\sum_{i=1}^{n} x_{i}\left|\tilde{\phi}_{i}\right\rangle\left\langle\tilde{\phi}_{i}\right| \geq 0
$$

This condition is equivalent to the positivity of the $n \times n$ matrix $M$ defined by

$$
\begin{aligned}
M_{i j} & =\left\langle\phi_{i}\left|\left(\mathbf{1}_{V}-\sum_{k=1}^{n} x_{k}\left|\tilde{\phi}_{k}\right\rangle\left\langle\tilde{\phi}_{k}\right|\right)\right| \phi_{j}\right\rangle \\
& =N_{i j}-x_{i} \delta_{i j}
\end{aligned}
$$

since $\left|\phi_{i}\right\rangle$ are linearly independent.

Thus, the unambiguous discrimination problem of the ensemble $\left\{\eta_{i},\left|\phi_{i}\right\rangle\right\}_{i=1}^{n}$ is formulated as semidefinite programming: The problem is to maximize

$$
p=\sum_{i=1}^{n} \eta_{i} x_{i}
$$

subject to conditions given by

$$
\begin{aligned}
& x_{i} \geq 0 \quad(i=1,2, \ldots, n), \\
& N-X \geq 0,
\end{aligned}
$$

where $X=\operatorname{diag}\left(x_{1}, x_{2}, \ldots, x_{n}\right)$ and variables are $x_{i}$. We can show that solutions $x_{i}$ are uniquely determined; the optimal measurement is unique. The proof is presented in the appendix.

\section{B. Relaxed problem and reduction of the number of states}

In unambiguous discrimination, the optimal strategy may produce a vanishing probability of identifying the input state with some of the states $\left|\phi_{i}\right\rangle$ : Some of $x_{i}$ may be zero in solutions of the problem (9). To see when this happens, it is convenient to consider the problem where the conditions (9b), $x_{i} \geq 0$, are omitted. To distinguish the two problems, this relaxed form of the problem is called a "relaxed $n$-state problem" from now on, and the original one (9) is simply called an " $n$-state problem."

In the relaxed $n$-state problem, the task is to maximize

$$
p^{\mathrm{R}}=\sum_{i=1}^{n} \eta_{i} x_{i}^{\mathrm{R}}
$$

subject to conditions given by

$$
N-X^{\mathrm{R}} \geq 0
$$

where $X^{\mathrm{R}}=\operatorname{diag}\left(x_{1}^{\mathrm{R}}, x_{2}^{\mathrm{R}}, \ldots, x_{n}^{\mathrm{R}}\right)$. We use a superscript $\mathrm{R}$ to represent quantities in the relaxed problem.

There are important relations between solutions of the two problems. Let $\boldsymbol{x}^{\mathrm{R}}=\left(x_{1}^{\mathrm{R}}, x_{2}^{\mathrm{R}}, \ldots, x_{n}^{\mathrm{R}}\right)$ be a solution of the relaxed $n$-state problem. Then we can show the following properties:

(I) If $x_{i}^{\mathrm{R}} \geq 0$ for all $i$, then $\boldsymbol{x}^{\mathrm{R}}$ is the solution of the $n$-state problem.

(II) If $x_{i}^{\mathrm{R}}<0$ for $i$ in some nonempty index set $S$, then the solution of the $n$-state problem has vanishing components, $x_{i}=0$, for at least one $i$ in $S$.

Property (I) is evident. To show property (II), we consider a linear interpolation between the two solutions as

$$
\hat{\boldsymbol{x}}(\kappa)=(1-\kappa) \boldsymbol{x}+\kappa \boldsymbol{x}^{\mathrm{R}}, \quad 0 \leq \kappa \leq 1,
$$




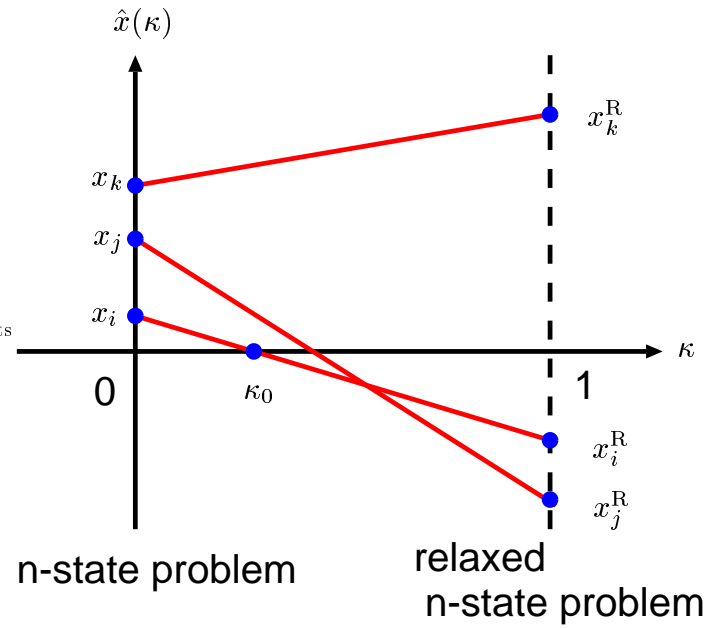

FIG. 1: (Color online) Linear interpolation of the solutions of the $n$-state problem and the relaxed $n$-state problem. Each component of $\hat{\boldsymbol{x}}(\kappa)$ is plotted vs. $\kappa$. There exists $0 \leq \kappa_{0}<1$ such that $\hat{x}_{k}\left(\kappa_{0}\right) \geq 0$ for all $k$ and $\hat{x}_{i}\left(\kappa_{0}\right)=0$ for a certain $i \in$ $S$. By the uniqueness of the solution of the $n$-state problem, $\kappa_{0}$ is actually 0 .

where $\boldsymbol{x}$ is the solution of the $n$-state problem and $\boldsymbol{x}^{\mathrm{R}}$ is a solution of the relaxed $n$-state problem. We find that $\hat{\boldsymbol{x}}(\kappa)$ satisfies the condition (9c) since

$$
N-\hat{X}(\kappa)=(1-\kappa)(N-X)+\kappa\left(N-X^{\mathrm{R}}\right) \geq 0 .
$$

The success probability corresponding to $\hat{\boldsymbol{x}}(\kappa)$ is written as

$$
\hat{p}(\kappa)=(1-\kappa) p_{\max }+\kappa p_{\max }^{\mathrm{R}},
$$

where $p_{\max }$ and $p_{\max }^{\mathrm{R}}$ are the maximum success probabilities of the $n$-state and relaxed $n$-state problems, respectively. Note that $p_{\max }^{\mathrm{R}} \geq p_{\max }$ by definition. From this we note that $\hat{p}(\kappa)$ is no less than $p_{\max }$ for $0 \leq \kappa \leq 1$. This implies that $\hat{\boldsymbol{x}}(\kappa)$ is a solution of the $n$-state problem if its all components $\hat{x}_{i}(\kappa)$ are nonnegative.

Obviously, $\hat{x}_{i}(\kappa)$ is continuous with respect to $\kappa$. We also know that $x_{i} \geq 0$ for all $i$ and that $x_{i}^{\mathrm{R}}<0$ for $i \in S$, whereas $x_{i}^{\mathrm{R}} \geq 0$ otherwise. Using the intermediate value theorem, we observe that there exists $0 \leq \kappa_{0}<1$ such that $\hat{x}_{k}\left(\kappa_{0}\right) \geq 0$ for all $k$ and $\hat{x}_{i}\left(\kappa_{0}\right)=\overline{0}$ for a certain $i \in S$ (see Fig. 11). Therefore, $\hat{\boldsymbol{x}}\left(\kappa_{0}\right)$ is a solution for the $n$-state problem. Remember that the solution of the $n$-state problem is unique, implying $\kappa_{0}=0$. Thus, we can conclude that the solution of the $n$-state problem has vanishing components, $x_{i}=0$, for at least one $i$ in $S$. This completes the proof of property (II).

Next, we show that if some of $x_{i}^{\mathrm{R}}$ are negative in a solution of the relaxed $n$-state problem, the $n$-state problem can be reduced to a certain $(n-1)$-state problem. Let us first assume that only one component is negative. Without loss of generality, we assume that $x_{n}^{\mathrm{R}}<0$ and the others are non-negative. Property (II) then states that the component $x_{n}$ is 0 in the solution of the $n$-state problem.
This solution can be obtained by requiring an additional constraint, $x_{n}=0$, to the original $n$-state problem. With this additional constraint, the condition (7) becomes

$$
\mathbf{1}_{V}-\sum_{i=1}^{n-1} x_{i}\left|\tilde{\phi}_{i}\right\rangle\left\langle\tilde{\phi}_{i}\right| \geq 0 .
$$

Let us define the following two sets of $n-1$ states:

$$
\begin{aligned}
\left|\phi_{i}^{\prime}\right\rangle & \equiv \frac{Q\left|\phi_{i}\right\rangle}{\sqrt{\left\langle\phi_{i}|Q| \phi_{i}\right\rangle}}(i=1,2, \ldots, n-1), \\
\left|\tilde{\phi}_{i}^{\prime}\right\rangle & \equiv \sqrt{\left\langle\phi_{i}|Q| \phi_{i}\right\rangle}\left|\tilde{\phi}_{i}\right\rangle \quad(i=1,2, \ldots, n-1),
\end{aligned}
$$

where

$$
Q \equiv \mathbf{1}_{V}-\left|\phi_{n}\right\rangle\left\langle\phi_{n}\right|
$$

is the projector onto the $(n-1)$-dimensional subspace $V^{\prime}$ spanned by the $n-1$ states $\left\{\left|\tilde{\phi}_{i}\right\rangle\right\}_{i=1}^{n-1}$.

It is readily verified that the two sets of states are reciprocal in the sense that $\left\langle\tilde{\phi}_{i}^{\prime} \mid \phi_{j}^{\prime}\right\rangle=\delta_{i j}$. Furthermore, the condition (14) can be expressed as

$$
\mathbf{1}_{V^{\prime}}-\sum_{i=1}^{n-1} x_{i}^{\prime}\left|\tilde{\phi}_{i}^{\prime}\right\rangle\left\langle\tilde{\phi}_{i}^{\prime}\right| \geq 0
$$

where $x_{i}^{\prime}$ is defined as

$$
x_{i}^{\prime} \equiv \frac{x_{i}}{\left\langle\phi_{i}|Q| \phi_{i}\right\rangle} .
$$

We will shortly see that $x_{i}^{\prime}$ are variables in the reduced $(n-1)$-state problem. The success probability of the $n$ state problem with the additional constraint, $x_{n}=0$, is rewritten as

$$
\begin{aligned}
p & =\sum_{i=1}^{n-1} \eta_{i} x_{i} \\
& =\left(\sum_{k=1}^{n-1} \eta_{k}\left\langle\phi_{k}|Q| \phi_{k}\right\rangle\right)\left(\sum_{i=1}^{n-1} \eta_{i}^{\prime} x_{i}^{\prime}\right),
\end{aligned}
$$

where $\eta_{i}^{\prime}$ is defined as

$$
\eta_{i}^{\prime} \equiv \frac{\eta_{i}\left\langle\phi_{i}|Q| \phi_{i}\right\rangle}{\sum_{k=1}^{n-1} \eta_{k}\left\langle\phi_{k}|Q| \phi_{k}\right\rangle} .
$$

Note that $\eta_{i}^{\prime}>0$ and $\sum_{i=1}^{n-1} \eta_{i}^{\prime}=1$. The $\eta_{i}^{\prime}$ turn out to be the occurrence probabilities in the $(n-1)$-state problem. From Eq. (20) and the condition (18), we observe that the $n$-state problem with the additional constraint $x_{n}=0$ is equivalent to the $(n-1)$-state problem for the ensemble $\left\{\eta_{i}^{\prime},\left|\phi_{i}^{\prime}\right\rangle\right\}_{i=1}^{n-1}$. The maximum success probabilities and the variables $x_{i}$ of the two problems are related in the following way:

$$
\begin{aligned}
& p_{\max }^{(n)}\left[\left\{\eta_{i},\left|\phi_{i}\right\rangle\right\}_{i=1}^{n}\right] \\
& =\left(\sum_{k=1}^{n-1} \eta_{k}\left\langle\phi_{k}|Q| \phi_{k}\right\rangle\right) p_{\max }^{(n-1)}\left[\left\{\eta_{i}^{\prime},\left|\phi_{i}^{\prime}\right\rangle\right\}_{i=1}^{n-1}\right], \\
& x_{i}=\left\langle\phi_{i}|Q| \phi_{i}\right\rangle x_{i}^{\prime} .
\end{aligned}
$$


It should be emphasized that the extra condition $x_{n}=0$ does not imply that the state $\left|\phi_{n}\right\rangle$ can completely be neglected. This is because the no-error conditions (2) should be respected for all the states including $\left|\phi_{n}\right\rangle$. The states to be discriminated in the reduced $(n-1)$-state problem are not $\left\{\left|\phi_{i}\right\rangle\right\}_{i=1}^{n-1}$ but $\left\{\left|\phi_{i}^{\prime}\right\rangle\right\}_{i=1}^{n-1}$, which are the normalized states with their $\left|\phi_{n}\right\rangle$ component subtracted. As can be seen from (21), the occurrence probabilities are also modified by the factor of $\left\langle\phi_{i}|Q| \phi_{i}\right\rangle$.

So far, we assumed that only one component of $\boldsymbol{x}^{\mathrm{R}}$ is negative. If more than one component is negative, we do not know which $x_{i}(i \in S)$ vanishes in the $n$-state problem. Therefore, for each $i$ in $S$, we need to solve the corresponding $(n-1)$-state problem with $x_{i}$ being set 0 . From Eq. (22), we obtain a finite number of candidates for the maximum success probability of the $n$-state problem. Evidently, the one with the largest probability is the solution of the $n$-state problem.

We can show that the number of negative components of $\boldsymbol{x}^{\mathrm{R}}$ is $n-1$ at most. This can be seen in the following way: The Gram matrix $N$ is strictly positive definite since the states $\left\{\left|\phi_{i}\right\rangle\right\}_{i=1}^{n}$ are linearly independent. This implies that the condition $N-X^{\mathrm{R}} \geq 0$ is satisfied for a set of sufficiently small but positive $x_{i}^{\mathrm{R}}$, which leads to a positive success probability. Therefore, $\boldsymbol{x}^{\mathrm{R}}$ with all components negative cannot be a solution of the relaxed $n$-state problem.

\section{Dual problem of the relaxed problem}

In our method, we first solve the relaxed $n$-state problem defined in Eq. (10). If all $x_{i}^{\mathrm{R}}$ are non-negative, they give the solution of the $n$-state problem. As shown in the preceding section, if some of $x_{i}^{\mathrm{R}}$ are negative, the problem is reduced to a certain $(n-1)$-state problem. How, then, do we solve the relaxed $n$-state problem? We find it convenient to consider the dual problem of the relaxed $n$-state problem.

The task in the dual problem is to minimize

$$
d \equiv \operatorname{tr} Y N
$$

subject to the conditions given by

$$
Y \geq 0, Y_{i i}=\eta_{i}
$$

where the variable is an $n \times n$ Hermitian matrix $Y$. It is readily shown that the minimum $d_{\text {min }}$ gives an upper bound for the maximum $p_{\max }^{\mathrm{R}}$ in the relaxed $n$-state problem (10).

$$
\begin{aligned}
p_{\max }^{\mathrm{R}} & =\sum_{i=1}^{n} \eta_{i} x_{i}^{\mathrm{R}}=\sum_{n-1}^{n} Y_{i i} x_{i}^{\mathrm{R}} \\
& =\operatorname{tr} Y X^{\mathrm{R}}=\operatorname{tr} Y\left[N-\left(N-X^{\mathrm{R}}\right)\right] \\
& \leq \operatorname{tr} Y N=d_{\min }
\end{aligned}
$$

where the inequality in the last line follows from the inequality $\operatorname{tr} Y\left(N-X^{\mathrm{R}}\right) \geq 0$. Therefore, the set of conditions given by

$$
\left(N-X^{\mathrm{R}}\right) Y=0
$$

together with the conditions $10 \mathrm{~b}$ ) and $(24 \mathrm{~b})$ is a sufficient condition for $d_{\min }$ to be equal to $p_{\max }^{\mathrm{R}}$. In the next section, for the $n=2$ and $n=3$ cases, we first solve the dual problem (24) and then we explicitly verify that this set of conditions are satisfied. According to the general theory of semidefinite programming [28], it is guaranteed that the solution of the $n$-state problem can always be obtained in this way.

The variable matrix $Y$ in the dual problem is constrained by the condition 24b ). Any positive semidefinite matrix can be expressed as the Gram matrix of some set of vectors. Therefore, we can parametrize $Y$ satisfying the condition (24b) as

$$
Y_{i j}=\left\langle y_{i} \mid y_{j}\right\rangle
$$

where $\left|y_{i}\right\rangle$ are arbitrary vectors with ||$\left.y_{i}\right\rangle \mid=\sqrt{\eta_{i}}$. This convenient parametrization will be used in the following sections.

\section{THREE PURE STATES WITH REAL INNER PRODUCTS}

In our scheme, we solve the dual problem (24) to obtain the solution of the relaxed problem (10). If all $x_{i}^{\mathrm{R}}$ are non-negative, they give the solution of the original nonrelaxed problem (91). Otherwise, the problem is reduced to the one for $n-1$ states. To demonstrate how this scheme works, we first solve the problem of two pure states by our scheme. This provides a helpful guideline for the three-state problem which will be discussed next.

\section{A. Two pure states}

The task of the dual problem is to minimize

$$
d=\operatorname{tr} Y N
$$

subject to

$$
Y \geq 0, Y_{i i}=\eta_{i}
$$

where variable $Y$ is a $2 \times 2$ Hermitian matrix. By changing the phase of the state $\left|\phi_{1}\right\rangle$, we can assume that the mutual inner product is real and negative, $\left\langle\phi_{1} \mid \phi_{2}\right\rangle=$ $-\left|\left\langle\phi_{1} \mid \phi_{2}\right\rangle\right|$. Using the parametrization (27), we can write $d$ as

$$
d=1-\left(\left\langle y_{1} \mid y_{2}\right\rangle+\left\langle y_{2} \mid y_{1}\right\rangle\right)\left|\left\langle\phi_{1} \mid \phi_{2}\right\rangle\right|
$$


Applying the triangle inequality and Schwarz's inequality to the second term of Eq. (30), we have

$$
\begin{aligned}
\left|\left\langle y_{1} \mid y_{2}\right\rangle+\left\langle y_{2} \mid y_{1}\right\rangle\right| & \leq 2\left|\left\langle y_{1} \mid y_{2}\right\rangle\right| \\
& \leq 2 \sqrt{\left\langle y_{1} \mid y_{1}\right\rangle} \sqrt{\left\langle y_{2} \mid y_{2}\right\rangle} \\
& =2 \sqrt{\eta_{1} \eta_{2}},
\end{aligned}
$$

where $\left\langle y_{i} \mid y_{i}\right\rangle=\eta_{i}$ is used. By this inequality, we obtain the minimum of $d$ to be

$$
d_{\min }=1-2 \sqrt{\eta_{1} \eta_{2}}\left|\left\langle\phi_{1} \mid \phi_{2}\right\rangle\right|
$$

and the vectors $\left|y_{i}\right\rangle$ attaining the minimum can be taken to be the following one-dimensional vectors:

$$
\left|y_{1}\right\rangle=\sqrt{\eta_{1}},\left|y_{2}\right\rangle=\sqrt{\eta_{2}}
$$

Thus, $Y$ is given by

$$
Y=\left(\begin{array}{cc}
\eta_{1} & \sqrt{\eta_{1} \eta_{2}} \\
\sqrt{\eta_{2} \eta_{1}} & \eta_{2}
\end{array}\right)=\boldsymbol{\beta} \boldsymbol{\beta}^{\dagger}
$$

with $\boldsymbol{\beta}$ defined to be

$$
\boldsymbol{\beta} \equiv\left(\begin{array}{l}
\sqrt{\eta_{1}} \\
\sqrt{\eta_{2}}
\end{array}\right)
$$

The attainability condition is given by Eq. (26). Substituting $Y$ in Eq.(26) and multiplying this equation by $\boldsymbol{\beta}$ on the right, we obtain

$$
\left(N-X^{\mathrm{R}}\right) \boldsymbol{\beta}=0
$$

since $\boldsymbol{\beta}^{\dagger} \boldsymbol{\beta}=1$. By using the matrix representation, Eq. (33) is rewritten as

$$
\left(\begin{array}{cc}
1-x_{1}^{\mathrm{R}} & -\left|\left\langle\phi_{1} \mid \phi_{2}\right\rangle\right| \\
-\left|\left\langle\phi_{1} \mid \phi_{2}\right\rangle\right| & 1-x_{2}^{\mathrm{R}}
\end{array}\right)\left(\begin{array}{l}
\sqrt{\eta_{1}} \\
\sqrt{\eta_{2}}
\end{array}\right)=0 .
$$

From this equation, we immediately find that $x_{1}^{\mathrm{R}}$ and $x_{2}^{\mathrm{R}}$ are given by

$$
\begin{aligned}
& x_{1}^{\mathrm{R}}=1-\sqrt{\frac{\eta_{2}}{\eta_{1}}}\left|\left\langle\phi_{1} \mid \phi_{2}\right\rangle\right|, \\
& x_{2}^{\mathrm{R}}=1-\sqrt{\frac{\eta_{1}}{\eta_{2}}}\left|\left\langle\phi_{1} \mid \phi_{2}\right\rangle\right| .
\end{aligned}
$$

The $x_{i}^{\mathrm{R}}$ must further satisfy Eq. (10b): The eigenvalues of $N-X^{\mathrm{R}}$ should be non-negative. The characteristic equation of $N-X^{\mathrm{R}}$ is given by

$$
\lambda\left(\lambda-\frac{\left|\left\langle\phi_{1} \mid \phi_{2}\right\rangle\right|}{\sqrt{\eta_{1} \eta_{2}}}\right)=0
$$

which clearly shows that the two eigenvalues are nonnegative. Therefore, $x_{i}^{\mathrm{R}}$ is the solution of the relaxed two-state problem.
Thus, if $x_{i}^{\mathrm{R}}$ are all non-negative, they give the optimal solution of the two-state problem $x_{i}$ as

$$
\begin{aligned}
& x_{1}=1-\sqrt{\frac{\eta_{2}}{\eta_{1}}}\left|\left\langle\phi_{1} \mid \phi_{2}\right\rangle\right|, \\
& x_{2}=1-\sqrt{\frac{\eta_{1}}{\eta_{2}}}\left|\left\langle\phi_{1} \mid \phi_{2}\right\rangle\right|,
\end{aligned}
$$

and the maximum success probability $p_{\max }$ is given by $d_{\min }$,

$$
p_{\max }=1-2 \sqrt{\eta_{1} \eta_{2}}\left|\left\langle\phi_{1} \mid \phi_{2}\right\rangle\right| .
$$

If one of $x_{i}^{\mathrm{R}}$ is negative, however, omitting one of the states to be discriminated is optimal. Let us first assume $x_{2}^{\mathrm{R}}<0$, which happens when the occurrence probabilities and the inner product satisfy the following condition:

$$
\sqrt{\frac{\eta_{2}}{\eta_{1}}}<\left|\left\langle\phi_{1} \mid \phi_{2}\right\rangle\right|
$$

We then need to search for the optimal solution with $x_{2}=0$. According to Eqs. (15) and (21), the two-state problem with the additional constraint $x_{2}=0$ is equivalent to the one-state problem for the following state $\left|\phi_{1}^{\prime}\right\rangle$ with occurrence probability $\eta_{1}^{\prime}$ :

$$
\left|\phi_{1}^{\prime}\right\rangle=\frac{Q\left|\phi_{1}\right\rangle}{\sqrt{\left\langle\phi_{1}|Q| \phi_{1}\right\rangle}}, \quad \eta_{1}^{\prime}=\frac{\eta_{1}\left\langle\phi_{1}|Q| \phi_{1}\right\rangle}{\eta_{1}\left\langle\phi_{1}|Q| \phi_{1}\right\rangle}=1
$$

where $Q$ is the projector defined by $Q \equiv \mathbf{1}-\left|\phi_{2}\right\rangle\left\langle\phi_{2}\right|$. The maximum success probabilities and the variables $x_{i}$ and $x_{i}^{\prime}$ of the two problems are related as follows:

$$
\begin{aligned}
p_{\max }^{(2)}\left[\left\{\eta_{i},\left|\phi_{i}\right\rangle\right\}_{i=1}^{2}\right] & =\eta_{1}\left\langle\phi_{1}|Q| \phi_{1}\right\rangle p_{\max }^{(1)}\left[\left\{\eta_{1}^{\prime},\left|\phi_{1}^{\prime}\right\rangle\right\}\right], \\
x_{1} & =\left\langle\phi_{1}|Q| \phi_{1}\right\rangle x_{1}^{\prime}
\end{aligned}
$$

where $x_{1}^{\prime}$ is the solution of the one-state problem.

The one-state problem is trivial: $p_{\max }^{(1)}\left[\left\{\eta_{1}^{\prime},\left|\phi_{1}^{\prime}\right\rangle\right\}\right]=1$ and $x_{1}^{\prime}=1$, since there is only one state to be discriminated. Thus, the maximum success probability $p_{\max }$ is obtained to be

$$
p_{\max }=\eta_{1}\left(1-\left|\left\langle\phi_{1} \mid \phi_{2}\right\rangle\right|^{2}\right)
$$

and $x_{i}(i=1,2)$ is given by

$$
\begin{aligned}
& x_{1}=1-\left|\left\langle\phi_{1} \mid \phi_{2}\right\rangle\right|^{2}, \\
& x_{2}=0 .
\end{aligned}
$$

We assumed that $x_{2}^{\mathrm{R}}<0$. For the case of $x_{1}^{\mathrm{R}}<0$, it is clear that this problem becomes a similar two-state problem with the additional constraint $x_{1}=0$.

The maximum success probability and the optimal solution $x_{i}$ are summarized in the following way:

$$
\begin{aligned}
\text { (i) } \sqrt{\frac{\eta_{2}}{\eta_{1}}} & <\left|\left\langle\phi_{1} \mid \phi_{2}\right\rangle\right| \\
& p_{\max }=\eta_{1}\left(1-\left|\left\langle\phi_{1} \mid \phi_{2}\right\rangle\right|^{2}\right)
\end{aligned}
$$




$$
\begin{aligned}
& x_{1}=1-\left|\left\langle\phi_{1} \mid \phi_{2}\right\rangle\right|^{2}, \\
& x_{2}=0 .
\end{aligned}
$$

(ii) $\left|\left\langle\phi_{1} \mid \phi_{2}\right\rangle\right| \leq \sqrt{\frac{\eta_{2}}{\eta_{1}}} \leq \frac{1}{\left|\left\langle\phi_{1} \mid \phi_{2}\right\rangle\right|}$

$$
p_{\max }=1-2 \sqrt{\eta_{1} \eta_{2}}\left|\left\langle\phi_{1} \mid \phi_{2}\right\rangle\right|
$$

$$
\begin{aligned}
& x_{1}=1-\sqrt{\frac{\eta_{2}}{\eta_{1}}}\left|\left\langle\phi_{1} \mid \phi_{2}\right\rangle\right|, \\
& x_{2}=1-\sqrt{\frac{\eta_{1}}{\eta_{2}}}\left|\left\langle\phi_{1} \mid \phi_{2}\right\rangle\right| .
\end{aligned}
$$

(iii)

$$
\begin{aligned}
\frac{1}{\left|\left\langle\phi_{1} \mid \phi_{2}\right\rangle\right|}<\sqrt{\frac{\eta_{2}}{\eta_{1}}} & \\
p_{\max } & =\eta_{2}\left(1-\left|\left\langle\phi_{1} \mid \phi_{2}\right\rangle\right|^{2}\right), \\
x_{1} & =0 \\
x_{2} & =1-\left|\left\langle\phi_{1} \mid \phi_{2}\right\rangle\right|^{2}
\end{aligned}
$$

This reproduces the well-known result of the unambiguous discrimination problem between two pure states. These results will be used in the subsequent section.

\section{B. Three pure states}

We consider the unambiguous discrimination problem between three pure states, $\left|\phi_{1}\right\rangle,\left|\phi_{2}\right\rangle$, and $\left|\phi_{3}\right\rangle$, with occurrence probabilities $\eta_{1}, \eta_{2}$, and $\eta_{3}$, respectively.

The inner products between the states depend on phases of the states. However, $\Gamma$ defined by

$$
\Gamma \equiv\left\langle\phi_{1} \mid \phi_{2}\right\rangle\left\langle\phi_{2} \mid \phi_{3}\right\rangle\left\langle\phi_{3} \mid \phi_{1}\right\rangle,
$$

is invariant under any phase change of each state. A little thought shows that the phases of the three mutual inner products can be chosen to be the same by appropriate phase changes of the states.

Here, we assume that $\Gamma$ is real, implying that the three mutual inner products can be chosen to be real. We separately consider two cases of $\Gamma \leq 0$ and $\Gamma>0$. The case of complex $\Gamma$ will be discussed in the next section.

\section{Case of $\Gamma \leq 0$}

In the case of $\Gamma \leq 0$, the phases of the mutual inner products can be chosen to be real and negative. As we will see, we can proceed along the same lines as in the two-state problem. The $d$ is given by

$$
\begin{aligned}
d= & \operatorname{tr} Y N \\
=1 & -\left(\left\langle y_{1} \mid y_{2}\right\rangle+\left\langle y_{2} \mid y_{1}\right\rangle\right)\left|\left\langle\phi_{1} \mid \phi_{2}\right\rangle\right| \\
& -\left(\left\langle y_{2} \mid y_{3}\right\rangle+\left\langle y_{3} \mid y_{2}\right\rangle\right)\left|\left\langle\phi_{2} \mid \phi_{3}\right\rangle\right| \\
& -\left(\left\langle y_{3} \mid y_{1}\right\rangle+\left\langle y_{1} \mid y_{3}\right\rangle\right)\left|\left\langle\phi_{3} \mid \phi_{1}\right\rangle\right| .
\end{aligned}
$$

Using the triangle inequality and Schwarz's inequality as in the two-state case, we immediately obtain the minimum of $d$ to be

$$
\begin{aligned}
d_{\min }=1 & -2 \sqrt{\eta_{1} \eta_{2}}\left|\left\langle\phi_{1} \mid \phi_{2}\right\rangle\right|-2 \sqrt{\eta_{2} \eta_{3}}\left|\left\langle\phi_{2} \mid \phi_{3}\right\rangle\right| \\
& -2 \sqrt{\eta_{3} \eta_{1}}\left|\left\langle\phi_{3} \mid \phi_{1}\right\rangle\right|,
\end{aligned}
$$

and the vectors $\left|y_{i}\right\rangle$ that attain this lower bound are the following one-dimensional vectors:

$$
\left|y_{1}\right\rangle=\sqrt{\eta_{1}},\left|y_{2}\right\rangle=\sqrt{\eta_{2}},\left|y_{3}\right\rangle=\sqrt{\eta_{3}} .
$$

Thus, $Y$ can be expressed as

$$
Y=\boldsymbol{\beta} \boldsymbol{\beta}^{\dagger},
$$

where $\boldsymbol{\beta}$ is defined by

$$
\boldsymbol{\beta}=\left(\begin{array}{c}
\sqrt{\eta_{1}} \\
\sqrt{\eta_{2}} \\
\sqrt{\eta_{3}}
\end{array}\right)
$$

The attainability condition (26) again takes the form,

$$
\left(N-X^{\mathrm{R}}\right) \boldsymbol{\beta}=0 .
$$

since $\boldsymbol{\beta}^{\dagger} \boldsymbol{\beta}=1$. In the matrix representation, Eq. (51) is written as

$$
\left(\begin{array}{ccc}
1-x_{1}^{\mathrm{R}} & -\left|\left\langle\phi_{1} \mid \phi_{2}\right\rangle\right| & -\left|\left\langle\phi_{3} \mid \phi_{1}\right\rangle\right| \\
-\left|\left\langle\phi_{1} \mid \phi_{2}\right\rangle\right| & 1-x_{2}^{\mathrm{R}} & -\left|\left\langle\phi_{2} \mid \phi_{3}\right\rangle\right| \\
-\left|\left\langle\phi_{3} \mid \phi_{1}\right\rangle\right| & -\left|\left\langle\phi_{2} \mid \phi_{3}\right\rangle\right| & 1-x_{3}^{\mathrm{R}}
\end{array}\right)\left(\begin{array}{c}
\sqrt{\eta_{1}} \\
\sqrt{\eta_{2}} \\
\sqrt{\eta_{3}}
\end{array}\right)
$$

$=0$.

Solving this equation, we obtain $x_{1}^{\mathrm{R}}, x_{2}^{\mathrm{R}}$, and $x_{3}^{\mathrm{R}}$ to be

$$
\begin{aligned}
& x_{1}^{\mathrm{R}}=1-\frac{1}{\sqrt{\eta_{1}}}\left(\sqrt{\eta_{2}}\left|\left\langle\phi_{1} \mid \phi_{2}\right\rangle\right|+\sqrt{\eta_{3}}\left|\left\langle\phi_{3} \mid \phi_{1}\right\rangle\right|\right), \\
& x_{2}^{\mathrm{R}}=1-\frac{1}{\sqrt{\eta_{2}}}\left(\sqrt{\eta_{1}}\left|\left\langle\phi_{1} \mid \phi_{2}\right\rangle\right|+\sqrt{\eta_{3}}\left|\left\langle\phi_{2} \mid \phi_{3}\right\rangle\right|\right), \\
& x_{3}^{\mathrm{R}}=1-\frac{1}{\sqrt{\eta_{3}}}\left(\sqrt{\eta_{1}}\left|\left\langle\phi_{3} \mid \phi_{1}\right\rangle\right|+\sqrt{\eta_{2}}\left|\left\langle\phi_{2} \mid \phi_{3}\right\rangle\right|\right) .
\end{aligned}
$$

Now we check if $x_{i}^{\mathrm{R}}$ satisfy the condition (10b), that is, the positivity of $N-X^{\mathrm{R}}$. The attainability condition (51) shows that one of the eigenvalues of $N-X^{\mathrm{R}}$ is 0 . The remaining two eigenvalues are determined by the following quadratic equation:

$$
\lambda^{2}-a \lambda+b=0,
$$


where the coefficients $a$ and $b$ are given by

$$
\begin{aligned}
a= & \frac{\eta_{1}+\eta_{2}}{\sqrt{\eta_{1} \eta_{2}}}\left|\left\langle\phi_{1} \mid \phi_{2}\right\rangle\right|+\frac{\eta_{2}+\eta_{3}}{\sqrt{\eta_{2} \eta_{3}}}\left|\left\langle\phi_{2} \mid \phi_{3}\right\rangle\right| \\
& +\frac{\eta_{3}+\eta_{1}}{\sqrt{\eta_{3} \eta_{1}}}\left|\left\langle\phi_{3} \mid \phi_{1}\right\rangle\right|, \\
b= & \frac{\left|\left\langle\phi_{2} \mid \phi_{3}\right\rangle\left\langle\phi_{3} \mid \phi_{1}\right\rangle\right|}{\sqrt{\eta_{1} \eta_{2}}}+\frac{\left|\left\langle\phi_{1} \mid \phi_{2}\right\rangle\left\langle\phi_{3} \mid \phi_{1}\right\rangle\right|}{\sqrt{\eta_{2} \eta_{3}}} \\
& +\frac{\left|\left\langle\phi_{1} \mid \phi_{2}\right\rangle\left\langle\phi_{2} \mid \phi_{3}\right\rangle\right|}{\sqrt{\eta_{3} \eta_{1}}} .
\end{aligned}
$$

The two roots, the eigenvalues of the real symmetric matrix, must be real, and the quadratic formula shows that they are both non-negative since $a \geq 0$ and $b \geq 0$. Thus, the positivity of $N-X^{\mathrm{R}}$ is satisfied, and $x_{i}^{\mathrm{R}}$ is therefore the solution of the relaxed problem.

If $x_{i}^{\mathrm{R}}$ are all non-negative, $x_{i}^{\mathrm{R}}$ is also the optimal solution of the original three-state problem,

$$
\begin{aligned}
& x_{1}=1-\frac{1}{\sqrt{\eta_{1}}}\left(\sqrt{\eta_{2}}\left|\left\langle\phi_{1} \mid \phi_{2}\right\rangle\right|+\sqrt{\eta_{3}}\left|\left\langle\phi_{3} \mid \phi_{1}\right\rangle\right|\right), \\
& x_{2}=1-\frac{1}{\sqrt{\eta_{2}}}\left(\sqrt{\eta_{1}}\left|\left\langle\phi_{1} \mid \phi_{2}\right\rangle\right|+\sqrt{\eta_{3}}\left|\left\langle\phi_{2} \mid \phi_{3}\right\rangle\right|\right), \\
& x_{3}=1-\frac{1}{\sqrt{\eta_{3}}}\left(\sqrt{\eta_{1}}\left|\left\langle\phi_{3} \mid \phi_{1}\right\rangle\right|+\sqrt{\eta_{2}}\left|\left\langle\phi_{2} \mid \phi_{3}\right\rangle\right|\right),
\end{aligned}
$$

and the optimal success probability $p_{\max }$ is given by $d_{\min }$ as follows:

$$
\begin{aligned}
p_{\max }=1 & -2 \sqrt{\eta_{1} \eta_{2}}\left|\left\langle\phi_{1} \mid \phi_{2}\right\rangle\right|-2 \sqrt{\eta_{2} \eta_{3}}\left|\left\langle\phi_{2} \mid \phi_{3}\right\rangle\right| \\
& -2 \sqrt{\eta_{3} \eta_{1}}\left|\left\langle\phi_{3} \mid \phi_{1}\right\rangle\right| .
\end{aligned}
$$

If some of $x_{i}^{\mathrm{R}}$ are negative, the problem can be reduced to a two-state problem. The two-state ensemble $\left\{\eta_{i}^{\prime},\left|\phi_{i}^{\prime}\right\rangle\right\}_{i=1}^{2}$ to be considered is determined by general formulas of Eqs. (15) and (21). The maximum success probability and the solution $x_{i}$ of the original problem can be obtained by Eqs. (22) and (23) in terms of the well-known solutions of two-state problems.

\section{Case of $\Gamma>0$}

In the case of $\Gamma>0$, the phases of the mutual inner products can be chosen to be real and positive. We introduce three positive real numbers $\alpha_{1}, \alpha_{2}$, and $\alpha_{3}$ as

$$
\begin{aligned}
& \alpha_{1} \equiv \sqrt{\frac{\left\langle\phi_{1} \mid \phi_{2}\right\rangle\left\langle\phi_{3} \mid \phi_{1}\right\rangle}{\left\langle\phi_{2} \mid \phi_{3}\right\rangle}}, \\
& \alpha_{2} \equiv \sqrt{\frac{\left\langle\phi_{1} \mid \phi_{2}\right\rangle\left\langle\phi_{2} \mid \phi_{3}\right\rangle}{\left\langle\phi_{3} \mid \phi_{1}\right\rangle}} \\
& \alpha_{3} \equiv \sqrt{\frac{\left\langle\phi_{2} \mid \phi_{3}\right\rangle\left\langle\phi_{3} \mid \phi_{1}\right\rangle}{\left\langle\phi_{1} \mid \phi_{2}\right\rangle}} .
\end{aligned}
$$

By using $\alpha_{i}$, the inner products can be expressed as follows:

$$
\left\langle\phi_{1} \mid \phi_{2}\right\rangle=\alpha_{1} \alpha_{2},\left\langle\phi_{2} \mid \phi_{3}\right\rangle=\alpha_{2} \alpha_{3},\left\langle\phi_{3} \mid \phi_{1}\right\rangle=\alpha_{3} \alpha_{1} .
$$

The $d$ can then be rewritten as

$$
\begin{aligned}
d & =\sum_{i, j=1}^{3} Y_{i j} N_{j i} \\
& =1+\sum_{i \neq j}^{3} \alpha_{i} \alpha_{j}\left\langle y_{i} \mid y_{j}\right\rangle \\
& \left.=1-\sum_{i=1}^{3} \eta_{i} \alpha_{i}^{2}+\left|\sum_{i=1}^{3} \alpha_{i}\right| y_{i}\right\rangle\left.\right|^{2} .
\end{aligned}
$$

Now, we must minimize $d$. This means that we must minimize $\left.\left|\sum_{i=1}^{3} \alpha_{i}\right| y_{i}\right\rangle \mid$, which is the norm of the sum of three vectors $\alpha_{1}\left|y_{1}\right\rangle, \alpha_{2}\left|y_{2}\right\rangle$, and $\alpha_{3}\left|y_{3}\right\rangle$. Here $\left|y_{i}\right\rangle$ is any vector with the norm $\sqrt{\eta_{i}}$. The minimum value of $\left.\left|\sum_{i=1}^{3} \alpha_{i}\right| y_{i}\right\rangle \mid$ is determined depending on whether the three lengths $\alpha_{i} \sqrt{\eta_{i}}$ satisfy the following set of relations:

$$
\begin{aligned}
& \alpha_{1} \sqrt{\eta_{1}} \leq \alpha_{2} \sqrt{\eta_{2}}+\alpha_{3} \sqrt{\eta_{3}} \\
& \alpha_{2} \sqrt{\eta_{2}} \leq \alpha_{1} \sqrt{\eta_{1}}+\alpha_{3} \sqrt{\eta_{3}} \\
& \alpha_{3} \sqrt{\eta_{3}} \leq \alpha_{1} \sqrt{\eta_{1}}+\alpha_{2} \sqrt{\eta_{2}}
\end{aligned}
$$

These relations are the condition for the three vectors $\alpha_{i}\left|y_{i}\right\rangle$ to form a triangle, and we call it the triangle condition hereafter.

First, we assume that the triangle condition (58) is satisfied. The minimum value of $\left.\left|\sum_{i=1}^{3} \alpha_{i}\right| y_{i}\right\rangle \mid$ is 0 , achieved by one-dimensional complex vector $\left|y_{i}\right\rangle=y_{i}$ with $\left|y_{i}\right|=\sqrt{\eta_{i}}$ such that $\alpha_{1} y_{1}+\alpha_{2} y_{2}+\alpha_{3} y_{3}=0$. The minimum value of $d$ is then given by

$$
d_{\min }=1-\sum_{i=1}^{3} \eta_{i} \alpha_{i}^{2}
$$

Writing

$$
\boldsymbol{y}=\left(\begin{array}{l}
y_{1} \\
y_{2} \\
y_{3}
\end{array}\right),
$$

we have $Y=\boldsymbol{y}^{*} \boldsymbol{y}^{T}$. Thus, Eq. (26) is rewritten as

$$
\left(N-X^{\mathrm{R}}\right) \boldsymbol{y}^{*}=0,
$$

from which we obtain the following three equations for $x_{1}^{\mathrm{R}}, x_{2}^{\mathrm{R}}$, and $x_{3}^{\mathrm{R}}$ :

$$
\begin{aligned}
& \left(1-x_{1}^{\mathrm{R}}\right) y_{1}^{*}+\alpha_{1} \alpha_{2} y_{2}^{*}+\alpha_{1} \alpha_{3} y_{3}^{*}=0 \\
& \alpha_{2} \alpha_{1} y_{1}^{*}+\left(1-x_{2}^{\mathrm{R}}\right) y_{2}^{*}+\alpha_{2} \alpha_{3} y_{3}^{*}=0 \\
& \alpha_{3} \alpha_{1} y_{1}^{*}+\alpha_{3} \alpha_{2} y_{2}^{*}+\left(1-x_{3}^{\mathrm{R}}\right) y_{3}^{*}=0 .
\end{aligned}
$$


Now, remember that $y_{i}$ are chosen in such a way that $\alpha_{1} y_{1}+\alpha_{2} y_{2}+\alpha_{3} y_{3}=0$. Thus, the first equation is given by

$$
\left(1-x_{1}^{\mathrm{R}}-\alpha_{1}^{2}\right) y_{1}^{*}=0 .
$$

Since $y_{1}^{*} \neq 0$, we obtain $x_{1}^{\mathrm{R}}=1-\alpha_{1}^{2}$. Similarly, we obtain $x_{2}^{\mathrm{R}}$ and $x_{3}^{\mathrm{R}}$.

$$
\begin{aligned}
& x_{1}^{\mathrm{R}}=1-\alpha_{1}^{2}, \\
& x_{2}^{\mathrm{R}}=1-\alpha_{2}^{2}, \\
& x_{3}^{\mathrm{R}}=1-\alpha_{3}^{2} .
\end{aligned}
$$

We can easily see the positivity of $N-X^{\mathrm{R}}$, since $N-$ $X^{\mathrm{R}}$ is expressed as $\boldsymbol{\alpha} \boldsymbol{\alpha}^{\dagger}$, where $\boldsymbol{\alpha}$ is defined by

$$
\boldsymbol{\alpha}=\left(\begin{array}{c}
\alpha_{1} \\
\alpha_{2} \\
\alpha_{3}
\end{array}\right)
$$

Thus, $x_{i}^{\mathrm{R}}$ we obtained is the optimal solution of the relaxed three-state problem.

If $x_{i}^{\mathrm{R}}$ are all non-negative, they are the optimal solution $x_{i}$ of the original three state problem,

$$
\begin{aligned}
& x_{1}=1-\alpha_{1}^{2}, \\
& x_{2}=1-\alpha_{2}^{2}, \\
& x_{3}=1-\alpha_{3}^{2} .
\end{aligned}
$$

and the success probability $p_{\max }$ is given by $d_{\min }$ as follows:

$$
p_{\max }=1-\left(\eta_{1} \alpha_{1}^{2}+\eta_{2} \alpha_{2}^{2}+\eta_{3} \alpha_{3}^{2}\right) .
$$

If some of $x_{i}^{\mathrm{R}}$ turn out to be negative, we can solve the problem by reducing it to the two-state problem as described before.

Next, we consider the case in which the triangle relation (58) is not satisfied. Comparing the norms of $\alpha_{1}\left|y_{1}\right\rangle, \alpha_{2}\left|y_{2}\right\rangle$, and $\alpha_{3}\left|y_{3}\right\rangle$, we realize that the norm $\left.\left|\sum_{i=1}^{3} \alpha_{i}\right| y_{i}\right\rangle \mid$ is minimized by subtracting the two smaller norms from the largest one. Suppose that $\alpha_{1} \sqrt{\eta_{1}} \geq \alpha_{2} \sqrt{\eta_{2}}, \alpha_{3} \sqrt{\eta_{3}}$. Then, the minimum value $d_{\min }$ is given by

$$
\begin{aligned}
& d_{\min }=1-\sum_{i=1}^{3} \eta_{i} \alpha_{i}^{2}+\left(\alpha_{1} \sqrt{\eta_{1}}-\alpha_{2} \sqrt{\eta_{2}}-\alpha_{3} \sqrt{\eta_{3}}\right)^{2} \\
& =1-2 \sqrt{\eta_{1} \eta_{2}} \alpha_{1} \alpha_{2}+2 \sqrt{\eta_{2} \eta_{3}} \alpha_{2} \alpha_{3}-2 \sqrt{\eta_{3} \eta_{1}} \alpha_{3} \alpha_{1}
\end{aligned}
$$

and the vectors $\left|y_{i}\right\rangle$ that attain this minimum value are the following one-dimensional vectors:

$$
\left|y_{1}\right\rangle=\sqrt{\eta_{1}},\left|y_{2}\right\rangle=-\sqrt{\eta_{2}},\left|y_{3}\right\rangle=-\sqrt{\eta_{3}} .
$$

Thus, $Y$ is given by $\boldsymbol{\beta} \boldsymbol{\beta}^{\dagger}$ with $\boldsymbol{\beta}$ defined to be

$$
\boldsymbol{\beta}=\left(\begin{array}{c}
\sqrt{\eta_{1}} \\
-\sqrt{\eta_{2}} \\
-\sqrt{\eta_{3}}
\end{array}\right)
$$

The rest of the argument proceeds in a quite similar way to that in the case of $\Gamma \leq 0$. Since $Y=\boldsymbol{\beta} \boldsymbol{\beta}^{\dagger}$, the attainability condition Eq. (26) can be rewritten as

$$
\left(N-X^{\mathrm{R}}\right) \boldsymbol{\beta}=0,
$$

with its matrix representation given by

$$
\left(\begin{array}{ccc}
1-x_{1}^{\mathrm{R}} & \alpha_{1} \alpha_{2} & \alpha_{1} \alpha_{3} \\
\alpha_{2} \alpha_{1} & 1-x_{2}^{\mathrm{R}} & \alpha_{2} \alpha_{3} \\
\alpha_{3} \alpha_{1} & \alpha_{3} \alpha_{2} & 1-x_{3}^{\mathrm{R}}
\end{array}\right)\left(\begin{array}{c}
\sqrt{\eta_{1}} \\
-\sqrt{\eta_{2}} \\
-\sqrt{\eta_{3}}
\end{array}\right)=0 .
$$

Solving this equation, we obtain $x_{i}^{\mathrm{R}}$ as follows:

$$
\begin{aligned}
& x_{1}^{\mathrm{R}}=1-\frac{\alpha_{1}}{\sqrt{\eta_{1}}}\left(\alpha_{2} \sqrt{\eta_{2}}+\alpha_{3} \sqrt{\eta_{3}}\right), \\
& x_{2}^{\mathrm{R}}=1-\frac{\alpha_{2}}{\sqrt{\eta_{2}}}\left(\alpha_{1} \sqrt{\eta_{1}}-\alpha_{3} \sqrt{\eta_{3}}\right), \\
& x_{3}^{\mathrm{R}}=1-\frac{\alpha_{3}}{\sqrt{\eta_{3}}}\left(\alpha_{1} \sqrt{\eta_{1}}-\alpha_{2} \sqrt{\eta_{2}}\right) .
\end{aligned}
$$

The positivity of $N-X^{\mathrm{R}}$ can be verified in the following way: One of the eigenvalues of $N-X^{\mathrm{R}}$ is 0 , which can be seen from Eq. (66). The remaining two eigenvalues are given by the roots of the following quadratic equation:

$$
\lambda^{2}-a \lambda+b=0
$$

where

$$
\begin{aligned}
a= & \frac{\alpha_{1}}{\sqrt{\eta_{1}}}\left(\alpha_{2} \sqrt{\eta_{2}}+\alpha_{3} \sqrt{\eta_{3}}\right)+\frac{\alpha_{2}}{\sqrt{\eta_{2}}}\left(\alpha_{1} \sqrt{\eta_{1}}-\alpha_{3} \sqrt{\eta_{3}}\right) \\
& +\frac{\alpha_{3}}{\sqrt{\eta_{3}}}\left(\alpha_{1} \sqrt{\eta_{1}}-\alpha_{2} \sqrt{\eta_{2}}\right) \\
b= & \frac{\alpha_{1} \alpha_{2} \alpha_{3}}{\sqrt{\eta_{1} \eta_{2} \eta_{3}}}\left(\alpha_{1} \sqrt{\eta_{1}}-\alpha_{2} \sqrt{\eta_{2}}-\alpha_{3} \sqrt{\eta_{3}}\right)
\end{aligned}
$$

Remember that we assumed that $\alpha_{1} \sqrt{\eta_{1}} \geq$ $\alpha_{2} \sqrt{\eta_{2}}, \alpha_{3} \sqrt{\eta_{3}}$, which implies that the relation (58a) is violated. Thus, we have $a \geq 0$ and $b \geq 0$. Therefore, the remaining two eigenvalues are also non-negative. Thus, $x_{i}^{\mathrm{R}}$ are the solution of the relaxed two-state problem.

If $x_{i}^{\mathrm{R}}$ are all non-negative, $x_{i}^{\mathrm{R}}$ is the optimal solution $x_{i}$ of the original three-state problem,

$$
\begin{aligned}
& x_{1}=1-\frac{1}{\sqrt{\eta_{1}}}\left(\sqrt{\eta_{2}}\left|\left\langle\phi_{1} \mid \phi_{2}\right\rangle\right|+\sqrt{\eta_{3}}\left|\left\langle\phi_{3} \mid \phi_{1}\right\rangle\right|\right), \\
& x_{2}=1-\frac{1}{\sqrt{\eta_{2}}}\left(\sqrt{\eta_{1}}\left|\left\langle\phi_{1} \mid \phi_{2}\right\rangle\right|-\sqrt{\eta_{3}}\left|\left\langle\phi_{2} \mid \phi_{3}\right\rangle\right|\right), \\
& x_{3}=1-\frac{1}{\sqrt{\eta_{3}}}\left(\sqrt{\eta_{1}}\left|\left\langle\phi_{3} \mid \phi_{1}\right\rangle\right|-\sqrt{\eta_{2}}\left|\left\langle\phi_{2} \mid \phi_{3}\right\rangle\right|\right),
\end{aligned}
$$

and the maximum success probability $p_{\max }$ is given by $d_{\min }$,

$$
\begin{aligned}
p_{\max }=1 & -2 \sqrt{\eta_{1} \eta_{2}}\left|\left\langle\phi_{1} \mid \phi_{2}\right\rangle\right|+2 \sqrt{\eta_{2} \eta_{3}}\left|\left\langle\phi_{2} \mid \phi_{3}\right\rangle\right| \\
& -2 \sqrt{\eta_{3} \eta_{1}}\left|\left\langle\phi_{3} \mid \phi_{1}\right\rangle\right| .
\end{aligned}
$$


Note that apparent asymmetry of the signs of terms in the above results is due to our assumption that $\alpha_{1} \sqrt{\eta_{1}} \geq$ $\alpha_{2} \sqrt{\eta_{2}}, \alpha_{3} \sqrt{\eta_{3}}$.

If some of $x_{i}^{\mathrm{R}}$ are negative, we can still have analytical results by considering the appropriate two-state problem in the same way as in other cases.

\section{Examples}

We consider the set of the following three-state vectors:

$$
\begin{aligned}
\left|\phi_{1}\right\rangle & =\left(\begin{array}{l}
1 \\
0 \\
0
\end{array}\right),\left|\phi_{2}\right\rangle=\left(\begin{array}{c}
\cos \varphi_{2} \\
\sin \varphi_{2} \\
0
\end{array}\right), \\
\left|\phi_{3}\right\rangle & =\left(\begin{array}{c}
\cos \varphi_{3} \sin \theta_{3} \\
\sin \varphi_{3} \sin \theta_{3} \\
\cos \theta_{3}
\end{array}\right) .
\end{aligned}
$$

In the first example, the equal occurrence probabilities are assumed, and $\varphi_{2}$ and $\varphi_{3}$ are fixed to be $\pi / 3$ and $\pi / 4$, respectively. Figure 2 displays the optimal solution $x_{i}$ and the maximum success probability as a function of $\theta_{3}$. As $\theta_{3}$ varies from 0 to $\pi / 2$, the type of solutions changes. When $\theta_{3}$ is small, all the three states have finite identification probabilities $x_{i}$. As $\theta_{3}$ increases, the number of the states with nonvanishing $x_{i}$ decreases. This reflects the fact that the state $\left|\phi_{3}\right\rangle$ approaches the state $\left|\phi_{2}\right\rangle$ and it becomes difficult to discriminate between these two states. When $\theta_{3}$ is in the range from $0.18(\mathrm{rad})$ to 1.01( $\mathrm{rad}), x_{1}$ and $x_{2}$ are constant. That is when the three lengths $\alpha_{i} \sqrt{\eta_{i}}$ satisfy the triangle condition (58) in the case of $\Gamma>0$. In such a case, $x_{i}$ is expressed only by the mutual inner products as in Eq. (63).

$$
\begin{aligned}
& x_{1}=1-\frac{\cos \varphi_{2} \cos \varphi_{3}}{\cos \left(\varphi_{2}-\varphi_{3}\right)}, \\
& x_{2}=1-\frac{\cos \varphi_{2} \cos \left(\varphi_{2}-\varphi_{3}\right)}{\cos \varphi_{3}}, \\
& x_{3}=1-\frac{\cos \varphi_{3} \cos \left(\varphi_{2}-\varphi_{3}\right)}{\cos \varphi_{2}} \sin ^{2} \theta_{3} .
\end{aligned}
$$

It is clear that $x_{1}$ and $x_{2}$ are independent of $\theta_{3}$. Thus, $x_{1}$ and $x_{2}$ are constant in this range.

In the second example, the occurrence probabilities are varied while the three states are fixed by setting $\varphi_{2}=\pi / 3, \varphi_{3}=\pi / 4$, and $\theta_{3}=\pi / 5$. Assuming $\eta_{1}=\eta_{2}$, we vary $\eta_{3}$ from 0 to 1 . Figure 3 displays the optimal solution $x_{i}$ and the maximum success probability as a function of $\eta_{3}$. For a sufficiently small $\eta_{3}$, the optimal solution gives $x_{3}=0$. As $\eta_{3}$ approaches 1 , only the state $\left|\phi_{3}\right\rangle$ survives as expected. However, the maximum success probability does not approach 1 . This is because we assumed $\eta_{i} \neq 0$ and imposed the no-error condition (2) for all states even if some of $\eta_{i}$ are very small. One interesting thing is that if $\Gamma>0$ and the triangle condition (58) is satisfied, Eq. (63) shows that $x_{i}$ does not depend on $\eta_{i}$ : The optimal measurement is independent of the
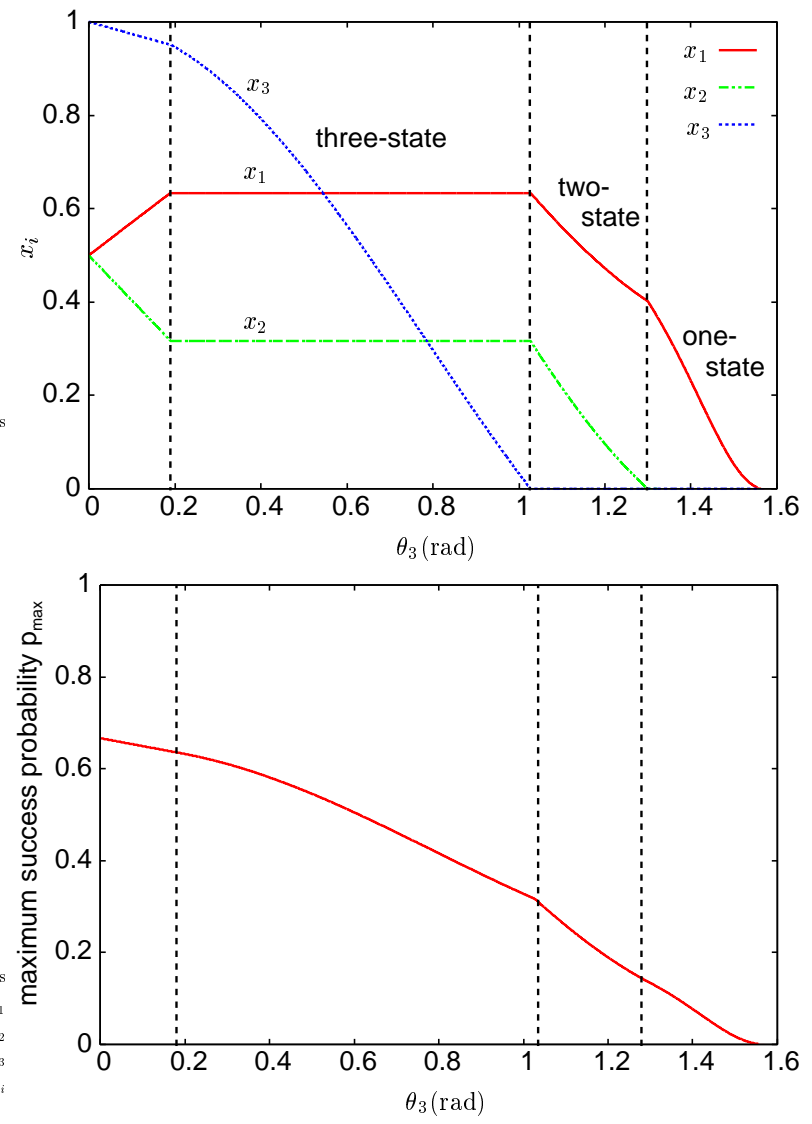

FIG. 2: (Color online) Example 1. $x_{i}$ (upper part) and the maximum success probability (lower part) vs. $\theta_{3}$. The angles $\varphi_{2}$ and $\varphi_{3}$ are fixed to be $\pi / 3$ and $\pi / 4$, respectively.

occurrence probabilities. This is the reason why all the $x_{i}$ are constant when $\eta_{3}$ ranges from 0.05 to 0.7 .

Before concluding the section, we mention that the problem of unambiguous discrimination can be numerically solved since the problem is formulated as semidefinite programming. We performed a numerical analysis for the above examples using the semidefinite programming package SDPA [29], and we compared the two results just to be sure. We verified that the numerical results reproduced our analytical ones within numerical errors.

\section{DISCUSSION}

In the preceding section, we have obtained complete analytical solutions for unambiguous discrimination of three pure states with real mutual inner products and general occurrence probabilities. Here, we examine what difficulties arise when the inner products are complex. Though our method is not applicable to general complex cases, we will show that there is one instance for which an analytical result can be obtained.

As in the preceding section, we define $\Gamma$ in the following 

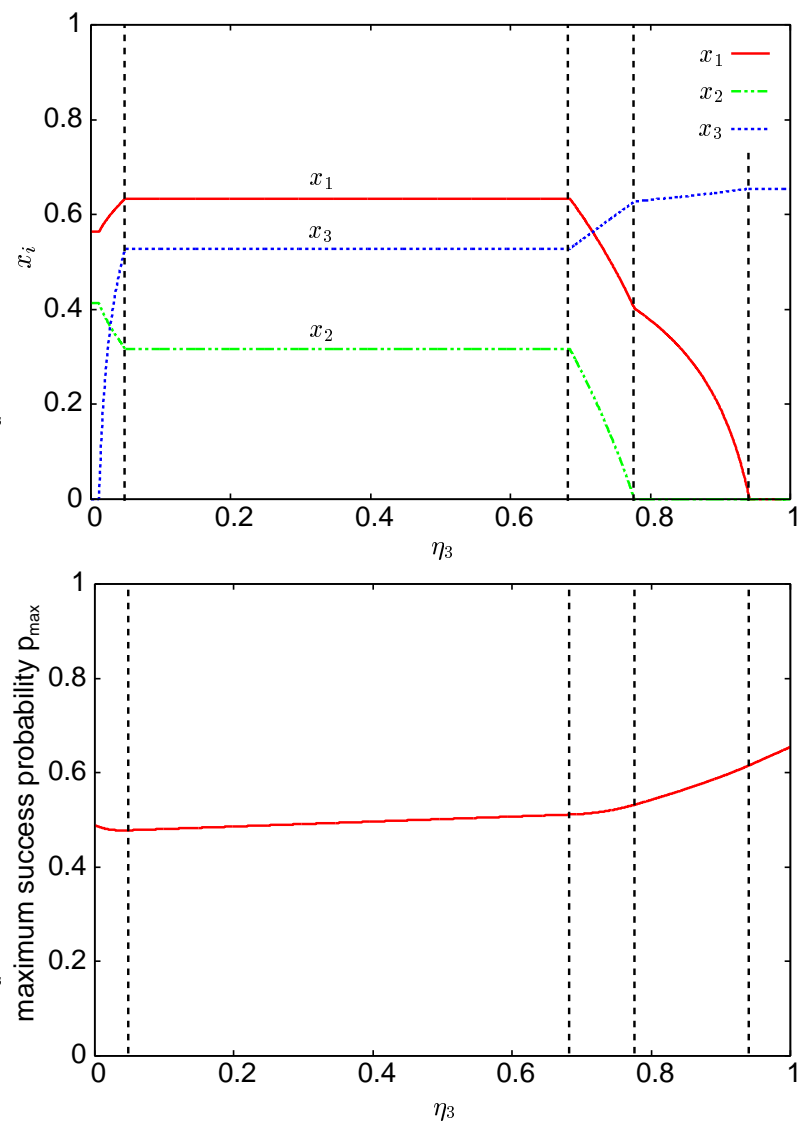

FIG. 3: (Color online) Example 2. $x_{i}$ (upper part) and the maximum success probability (lower part) vs. $\eta_{3} . \eta_{1}=\eta_{2}$ is assumed. The three states are fixed by setting $\varphi_{2}=\pi / 3$, $\varphi_{3}=\pi / 4$, and $\theta_{3}=\pi / 5$.

way:

$$
\Gamma=\left\langle\phi_{1} \mid \phi_{2}\right\rangle\left\langle\phi_{2} \mid \phi_{3}\right\rangle\left\langle\phi_{3} \mid \phi_{1}\right\rangle=|\Gamma| e^{3 i \theta}
$$

where $0 \leq \theta<2 \pi / 3$. We can evidently choose phases of the three states so that the phases of inner products $\left\langle\phi_{1} \mid \phi_{2}\right\rangle,\left\langle\phi_{2} \mid \phi_{3}\right\rangle$, and $\left\langle\phi_{3} \mid \phi_{1}\right\rangle$ are equal to the same value $\theta$. Then, we can express the three inner products in the following way:

$$
\begin{aligned}
& \left\langle\phi_{1} \mid \phi_{2}\right\rangle=\alpha_{1} \alpha_{2} e^{i \theta} \\
& \left\langle\phi_{2} \mid \phi_{3}\right\rangle=\alpha_{2} \alpha_{3} e^{i \theta} \\
& \left\langle\phi_{3} \mid \phi_{1}\right\rangle=\alpha_{3} \alpha_{1} e^{i \theta}
\end{aligned}
$$

where real positive numbers $\alpha_{i}$ are now defined in terms of the modulus of the inner products,

$$
\begin{aligned}
& \alpha_{1} \equiv \sqrt{\frac{\left|\left\langle\phi_{1} \mid \phi_{2}\right\rangle\right|\left|\left\langle\phi_{3} \mid \phi_{1}\right\rangle\right|}{\left|\left\langle\phi_{2} \mid \phi_{3}\right\rangle\right|}} \\
& \alpha_{2} \equiv \sqrt{\frac{\left|\left\langle\phi_{1} \mid \phi_{2}\right\rangle\right|\left|\left\langle\phi_{2} \mid \phi_{3}\right\rangle\right|}{\left|\left\langle\phi_{3} \mid \phi_{1}\right\rangle\right|}} \\
& \alpha_{3} \equiv \sqrt{\frac{\left|\left\langle\phi_{2} \mid \phi_{3}\right\rangle\right|\left|\left\langle\phi_{3} \mid \phi_{1}\right\rangle\right|}{\left|\left\langle\phi_{1} \mid \phi_{2}\right\rangle\right|}} .
\end{aligned}
$$

Writing $Y_{i j}=\left\langle y_{i} \mid y_{j}\right\rangle$ as before, we can express $d(=$ $\operatorname{tr} Y N)$ as follows:

$$
d=1+\langle\langle A|D| A\rangle\rangle
$$

where $|A\rangle\rangle$ is the three-component vector defined as

$$
|A\rangle\rangle=\left(\begin{array}{l}
\alpha_{1}\left|y_{1}\right\rangle \\
\alpha_{2}\left|y_{2}\right\rangle \\
\alpha_{3}\left|y_{3}\right\rangle
\end{array}\right)
$$

and $D$ is the $3 \times 3$ matrix given by

$$
D=\left(\begin{array}{ccc}
0 & e^{-i \theta} & e^{i \theta} \\
e^{i \theta} & 0 & e^{-i \theta} \\
e^{-i \theta} & e^{i \theta} & 0
\end{array}\right)
$$

The Hermitian matrix $D$ is readily diagonalized as

$$
U^{\dagger} D U=\operatorname{diag}\left(\lambda_{1}, \lambda_{2}, \lambda_{3}\right)
$$

by the unitary matrix given by

$$
U=\frac{1}{\sqrt{3}}\left(\begin{array}{ccc}
1 & 1 & 1 \\
1 & \omega & \omega^{2} \\
1 & \omega^{2} & \omega
\end{array}\right),
$$

where $\omega \equiv e^{i \frac{2}{3} \pi}$ is the cubic root of unity. The eigenvalues of $D$ are found to be

$$
\begin{aligned}
& \lambda_{1} \equiv 2 \cos \theta, \quad \lambda_{2} \equiv 2 \cos (\theta-2 \pi / 3), \\
& \lambda_{3} \equiv 2 \cos (\theta+2 \pi / 3) .
\end{aligned}
$$

We can now express $d$ of Eq. (176) in the following form:

$$
\left.\left.\left.d=1+\left.\frac{1}{3}\left(\lambda_{1}|| a_{1}\right\rangle\right|^{2}+\lambda_{2}|| a_{2}\right\rangle\left.\right|^{2}+\lambda_{3}|| a_{3}\right\rangle\left.\right|^{2}\right),
$$

where the three vectors $\left|a_{i}\right\rangle$ are defined by

$$
\begin{aligned}
& \left.\left(\begin{array}{l}
\left|a_{1}\right\rangle \\
\left|a_{2}\right\rangle \\
\left|a_{3}\right\rangle
\end{array}\right)=\sqrt{3} U^{\dagger}|A\rangle\right\rangle \\
& =\left(\begin{array}{c}
\alpha_{1}\left|y_{1}\right\rangle+\alpha_{2}\left|y_{2}\right\rangle+\alpha_{3}\left|y_{3}\right\rangle \\
\alpha_{1}\left|y_{1}\right\rangle+\omega^{2} \alpha_{2}\left|y_{2}\right\rangle+\omega \alpha_{3}\left|y_{3}\right\rangle \\
\alpha_{1}\left|y_{1}\right\rangle+\omega \alpha_{2}\left|y_{2}\right\rangle+\omega^{2} \alpha_{3}\left|y_{3}\right\rangle
\end{array}\right) .
\end{aligned}
$$

Equation (82) shows that $d$ is a linear combination of squared norms of the three vectors $\left|a_{i}\right\rangle$. Remember that 
$\left|y_{i}\right\rangle$ is arbitrary with only one constraint on its norm, ||$\left.y_{i}\right\rangle \mid=\sqrt{\eta_{i}}$. The maximum and minimum of the norm of each $\left|a_{i}\right\rangle$ can easily be obtained: The minimum of ||$\left.a_{i}\right\rangle \mid$ is 0 if the three lengths $\alpha_{i} \sqrt{\eta_{i}}$ satisfy the triangle condition (58). Otherwise, the minimum is given by $\alpha_{1} \sqrt{\eta_{1}}-\alpha_{2} \sqrt{\eta_{2}}-\alpha_{3} \sqrt{\eta_{3}}$, where we assume $\alpha_{1} \sqrt{\eta_{1}}$ is the greatest of all $\alpha_{i} \sqrt{\eta_{i}}$. The maximum of ||$\left.a_{i}\right\rangle \mid$ is evidently given by $\alpha_{1} \sqrt{\eta_{1}}+\alpha_{2} \sqrt{\eta_{2}}+\alpha_{3} \sqrt{\eta_{3}}$. We also notice that the sum of three squared norms of $\left|a_{i}\right\rangle$ is constant.

$$
\left.\sum_{i=1}^{3}|| a_{i}\right\rangle\left.\right|^{2}=3\langle\langle A \mid A\rangle\rangle=3 \sum_{i=1}^{3} \alpha_{i}^{2} \eta_{i} .
$$

Using this identity, we can rewrite $d$ in terms of any pair of squared norms of $\left|a_{i}\right\rangle$, which is still difficult to analytically minimize. However, if two of the eigenvalues $\lambda_{i}$ are equal, $d$ can be expressed by the squared norm of a single vector $\left|a_{i}\right\rangle$, which can be minimized very easily. We find that this occurs if and only if $\theta=0$ or $\theta=\pi / 3$. Clearly, these two cases correspond to the case of real $\Gamma$, which was solved in the preceding section. It can be verified that we obtain the same results as before in these cases.

There is one instance for which we can easily obtain an analytical result even if $\Gamma$ is complex. This is the case in which three $\alpha_{i} \sqrt{\eta_{i}}$ are equal to each other. Eliminating ||$\left.a_{3}\right\rangle \mid$ by using Eq. (84), we have

$$
\begin{aligned}
d=1 & +\lambda_{3} \sum_{i=1}^{3} \alpha_{i}^{2} \eta_{i} \\
& \left.\left.+\left.\frac{1}{3}\left(\left(\lambda_{1}-\lambda_{3}\right)|| a_{1}\right\rangle\right|^{2}+\left(\lambda_{2}-\lambda_{3}\right)|| a_{2}\right\rangle\left.\right|^{2}\right)
\end{aligned}
$$

We find that the two norms ||$\left.a_{1}\right\rangle \mid$ and ||$\left.a_{2}\right\rangle \mid$ can simultaneously be 0 if $\alpha_{i} \sqrt{\eta_{i}}$ is constant. This is possible by choosing $\left|y_{i}\right\rangle$, for example, to be single-component complex vectors so that three $\alpha_{i}\left|y_{i}\right\rangle$ make an equilateral triangle in the complex plane.

$$
\left|y_{1}\right\rangle=\sqrt{\eta_{1}},\left|y_{2}\right\rangle=\sqrt{\eta_{2}} \omega^{2},\left|y_{3}\right\rangle=\sqrt{\eta_{3}} \omega
$$

Note that $\lambda_{1} \geq \lambda_{3}$ and $\lambda_{2} \geq \lambda_{3}$. Thus, the minimum of $d$ is given by

$$
d_{\min }=1+2 \cos \left(\theta+\frac{2}{3} \pi\right) \sum_{i=1}^{3} \alpha_{i}^{2} \eta_{i} .
$$

The attainability condition (26) requires that

$$
x_{i}^{\mathrm{R}}=1+2 \alpha_{i}^{2} \cos \left(\theta+\frac{2}{3} \pi\right)
$$

The positivity of $N-X^{\mathrm{R}}$ can be verified in the following way:

$$
\begin{aligned}
& N-X^{\mathrm{R}} \\
& =\operatorname{diag}\left(\alpha_{1}, \alpha_{2}, \alpha_{3}\right)\left(D^{*}-\lambda_{3} \mathbf{1}\right) \operatorname{diag}\left(\alpha_{1}, \alpha_{2}, \alpha_{3}\right) \\
& \geq 0,
\end{aligned}
$$

where $D^{*}$ is the complex conjugate of $D$, and the inequality follows because $\lambda_{3}$ is also the smallest eigenvalue of $D^{*}$. Therefore, the set of $x_{i}^{\mathrm{R}}$ given by Eq. (88) is the solution of the relaxed problem. If all $x_{i}^{\mathrm{R}}$ are non-negative, they are also the solution of the nonrelaxed problem. The maximum success probability is then given by $d_{\min }$,

$$
p_{\max }=d_{\min }=1+2 \cos \left(\theta+\frac{2}{3} \pi\right) \sum_{i=1}^{3} \alpha_{i}^{2} \eta_{i} .
$$

Otherwise, the problem is reduced to a certain two-state problem.

This result is a generalization of the symmetric states considered in Ref. 11], though the number of states is limited to three here. For the symmetric states, the absolute values of all mutual inner products are the same, implying a constant $\alpha_{i}$, and the occurrence probabilities $\eta_{i}$ are assumed equal. In this case, $x_{i}^{\mathrm{R}}$ are shown to be the same and nonnegative, and the maximal success probability is given by

$$
p_{\max }=1+2 \gamma \cos \left(\theta+\frac{2}{3} \pi\right),
$$

where $\gamma=\left|\left\langle\phi_{1} \mid \phi_{2}\right\rangle\right|=\left|\left\langle\phi_{2} \mid \phi_{3}\right\rangle\right|=\left|\left\langle\phi_{3} \mid \phi_{1}\right\rangle\right|$.

\section{SUMMARY AND CONCLUDING REMARKS}

We have established some general properties of unambiguous discrimination of $n$ linearly independent pure states. By formulating the problem as semidefinite programming, we have shown that the solution of the problem is unique. In unambiguous discrimination, the optimal strategy may produce a vanishing probability of identifying the input state with some of the states. To deal with this possibility, it has been shown to be very convenient to consider a problem in which some constraints on the variables are relaxed. Applying our method to the case of three pure states, we have obtained complete analytic solutions if their mutual inner products are real. Unfortunately, our method is not generally applicable to the case of complex inner products. However, we have found a class of ensemble of three pure states with complex inner products for which we obtain an analytical solution.

It will also be of interest in future studies to extend our method to discrimination of three pure states with error margin [9, 10] and to discrimination of three unitary processes [30].

\section{Appendix: Uniqueness of solutions in the $n$-state problem}

We will show the solution of the $n$-state problem is uniquely determined. The following two propositions hold: 
Proposition 1. If $\boldsymbol{x}$ and $\boldsymbol{x}^{\prime}$ are both solutions of the $n$-state problem, then $x_{i_{0}}=x_{i_{0}}^{\prime}$ for at least one $i_{0}$.

Proposition 2. Consider the n-state problem with an additional condition: One of the components $x_{i_{0}}$ is fixed to be some value. We assume a solution exists. Then, the remaining variables are either 0 or uniquely determined by a certain $r$-state problem, where $1 \leq r \leq n-1$.

With these two propositions, the uniqueness of the solution of the $n$-state problem follows by induction on the number $n$ of the states. It is evident when $n=1$. We assume that the solution is unique in the $r$-state problem for $r \leq n-1$. Let $\boldsymbol{x}$ and $\boldsymbol{x}^{\prime}$ be two solutions of the $n$ state problem. By proposition (I), we have $x_{i_{0}}=x_{i_{0}}^{\prime}$ for a certain $i_{0}$. Proposition (II) states that the remaining variables are determined by a certain $r$-state problem, where $1 \leq r \leq n-1$. By the assumption, we can conclude $\boldsymbol{x}=\boldsymbol{x}^{\prime}$.

Now we present the proof of the two propositions.

Proof of Proposition 1. If $N-X>0$, then we can add a sufficiently small positive quantity to any component $x_{i}$ so the condition $N-X \geq 0$ is still satisfied while the success probability increases. Therefore, $\operatorname{det}(N-X)=0$ if $\boldsymbol{x}$ is a solution. Since a convex linear combination of the two solutions is also a solution, we have

$$
\operatorname{det}\left[N-\kappa X-(1-\kappa) X^{\prime}\right]=0 \quad(0 \leq \kappa \leq 1) .
$$

The coefficient of $\kappa^{n}$ of this determinant is given by

$$
\left(x_{1}^{\prime}-x_{1}\right)\left(x_{2}^{\prime}-x_{2}\right) \cdots\left(x_{n}^{\prime}-x_{n}\right),
$$

which should be 0 , implying $x_{i_{0}}=x_{i_{0}}^{\prime}$ for a certain $i_{0}$.

Proof of Proposition 2. Without loss of generality, we assume $i_{0}=n$. We write the condition (7) as

$$
\begin{aligned}
& \Delta-\sum_{i=1}^{n-1} x_{i}\left|\tilde{\phi}_{i}\right\rangle\left\langle\tilde{\phi}_{i}\right| \geq 0, \\
& \Delta \equiv \mathbf{1}_{V}-x_{n}\left|\tilde{\phi}_{n}\right\rangle\left\langle\tilde{\phi}_{n}\right| .
\end{aligned}
$$

The assumption that a solution exists to the problem implies $\Delta \geq 0$. We first consider the case $\Delta>0$. The condition (A.3) can be written as

$$
\mathbf{1}_{V}-\sum_{i=1}^{n-1} x_{i} \Delta^{-1 / 2}\left|\tilde{\phi}_{i}\right\rangle\left\langle\tilde{\phi}_{i}\right| \Delta^{-1 / 2} \geq 0 .
$$

The states $\left\{\Delta^{-1 / 2}\left|\tilde{\phi}_{i}\right\rangle\right\}_{i=1}^{n-1}$ are linearly independent. By $V^{\prime}$, we denote the $(n-1)$-dimensional subspace spanned by them. We define the following two sets of $n-1$ states in $V^{\prime}$ :

$$
\begin{aligned}
\left|\phi_{i}^{\prime}\right\rangle & =\frac{Q\left|\phi_{i}\right\rangle}{\sqrt{\left\langle\phi_{i}|Q| \phi_{i}\right\rangle}}(i=1, \ldots, n-1), \\
\left|\tilde{\phi}_{i}^{\prime}\right\rangle & =\sqrt{\left\langle\phi_{i}|Q| \phi_{i}\right\rangle} \Delta^{-1 / 2}\left|\tilde{\phi}_{i}\right\rangle \quad(i=1, \ldots, n-1),
\end{aligned}
$$

where $Q$ is the projector onto the subspace $V^{\prime}$. It can be readily verified that $\left\{\left|\phi_{i}^{\prime}\right\rangle\right\}_{i=1}^{n-1}$ and $\left\{\left|\tilde{\phi}_{i}^{\prime}\right\rangle\right\}_{i=1}^{n-1}$ are reciprocal in the sense that $\left\langle\tilde{\phi}_{i}^{\prime} \mid \phi_{j}^{\prime}\right\rangle=\delta_{i j}$. The condition (A.5) takes the form given by

$$
\mathbf{1}_{V^{\prime}}-\sum_{i=1}^{n-1} x_{i}^{\prime}\left|\tilde{\phi}_{i}^{\prime}\right\rangle\left\langle\tilde{\phi}_{i}^{\prime}\right| \geq 0
$$

where $x_{i}^{\prime} \equiv x_{i} /\left\langle\phi_{i}|Q| \phi_{i}\right\rangle$. The success probability to be maximized can be expressed as

$$
p=\sum_{i=1}^{n} \eta_{i} x_{i}=\eta_{n} x_{n}+N \sum_{i=1}^{n-1} \eta_{i}^{\prime} x_{i}^{\prime},
$$

where

$$
\begin{aligned}
\eta_{i}^{\prime} & \equiv \eta_{i}\left\langle\phi_{i}|Q| \phi_{i}\right\rangle / N, \\
N & \equiv \sum_{i=1}^{n-1} \eta_{i}\left\langle\phi_{i}|Q| \phi_{i}\right\rangle .
\end{aligned}
$$

Thus, maximization of $p$ in Eq. A.9 subject to the conditions A.8 and $x_{i}^{\prime}>0$ is equivalent to the $(n-1)$-state problem for the ensemble $\left\{\eta_{i}^{\prime},\left|\phi_{i}^{\prime}\right\rangle\right\}_{i=1}^{n-1}$.

Next, we consider the case in which $\Delta$ is singular. We observe that this happens if and only if $x_{n}=1 /\left\langle\tilde{\phi}_{n} \mid \tilde{\phi}_{n}\right\rangle$, and that $\Delta$ is the projector such that

$$
\begin{aligned}
& \Delta\left|\phi_{i}\right\rangle=\left|\phi_{i}\right\rangle \quad(i=1, \ldots, n-1), \\
& \Delta\left|\tilde{\phi}_{n}\right\rangle=0 .
\end{aligned}
$$

We also find that $x_{i}$ should be 0 unless $\left\langle\tilde{\phi}_{n} \mid \tilde{\phi}_{i}\right\rangle=0$. Without loss of generality, we assume that

$$
\left\langle\tilde{\phi}_{n} \mid \tilde{\phi}_{i}\right\rangle= \begin{cases}0 & i=1, \ldots, r \\ \text { nonzero } & i=r+1, \ldots, n-1,\end{cases}
$$

where $0 \leq r \leq n-1$. If $r=0$ then all $x_{i}$ are 0 . We assume $r \geq 1$. Let $\bar{V}_{r}^{\prime}$ be the subspace spanned by the linearly independent states $\left\{\left|\tilde{\phi}_{i}^{\prime}\right\rangle\right\}_{i=1}^{r}$. Since $V_{r}^{\prime}$ is evidently contained in the support of $\Delta$, the condition A.3 can be written as

$$
\mathbf{1}_{V_{r}^{\prime}}-\sum_{i=1}^{r} x_{i}\left|\tilde{\phi}_{i}\right\rangle\left\langle\tilde{\phi}_{i}\right| \geq 0
$$

The states which are reciprocal to the set $\left\{\left|\tilde{\phi}_{i}\right\rangle\right\}_{i=1}^{r}$ are not generally normalized. However, it is always possible to obtain the set of normalized states $\left\{\left|\phi_{i}^{\prime}\right\rangle\right\}_{i=1}^{r}$ which is reciprocal to the set $\left\{c_{i}\left|\tilde{\phi}_{i}\right\rangle\right\}_{i=1}^{r}$ by choosing the coefficients $c_{i}$ appropriately. Note that the resultant states $\left|\phi_{i}^{\prime}\right\rangle$ are unique up to a phase factor. Thus, maximizing the success probability subject to the conditions A.13 and $x_{i} \geq 0$ is equivalent to the $r$-state problem for the states $\left\{\left|\phi_{i}^{\prime}\right\rangle\right\}_{i=1}^{r}$ with properly modified occurrence probabilities $\eta_{i}^{\prime}$. 
[1] C. W. Helstrom, Quantum Detection and Estimation Theory (Academic Press, New York, 1976).

[2] A. S. Holevo, Probabilistic and Statistical Aspects of Quantum Theory (North-Holland, Amsterdam, 1982).

[3] A. Chefles, Contemp. Phys. 41, 401 (2000).

[4] I. D. Ivanovic, Phys. Lett. A 123, 257 (1987).

[5] D. Dieks, Phys. Lett. A 126, 303 (1988).

[6] A. Peres, Phys. Lett. A 128, 19 (1988).

[7] G. Jaeger and A. Shimony, Phys. Lett. A 197, 83 (1995).

[8] R. B. M. Clarke, A. Chefles, S. M. Barnett, and E. Riis, Phys. Rev. A 63, 040305 (2001).

[9] A. Hayashi, T. Hashimoto, and M. Horibe, Phys. Rev. A 78, 012333 (2008).

[10] H. Sugimoto, T. Hashimoto, M. Horibe, and A. Hayashi, Phys. Rev. A 80, 052322, (2009).

[11] A. Chefles, Phys. Lett. A 239, 339 (1998).

[12] A. Chefles and S. M. Barnett, Phys. Lett. A 250, 223 (1998).

[13] A. Peres and D. Terno, J. Phys. A 31, 7105 (1998).

[14] Y. Sun, M. Hillery, and J. A. Bergou, Phys. Rev. A 64, 022311 (2001).

[15] S. Zhang, Y. Feng, X. Sun, and M. Ying, Phys. Rev. A 64, 062103 (2001).

[16] Y. C. Eldar, IEEE Trans. Inf. Theory 49, 446 (2003).

[17] M. A. Jafarizadeh, M. Rezaei, N. Karimi, and
A. R. Amiri, Phys. Rev. A 77, 042314 (2008).

[18] B. F. Samsonov, Phys. Rev. A 79, 042312 (2009).

[19] S. Pang and S. Wu, Phys. Rev. A 80, 052320 (2009).

[20] T. Rudolph, R. W. Spekkens, and P. S. Turner, Phys. Rev. A 68, 010301 (2003).

[21] P. Raynal, N. Lutkenhaus, and S. J. van Enk, Phys. Rev. A 68, 022308 (2003).

[22] Y. C. Eldar, M. Stojnic, and B. Hassibi, Phys. Rev. A 69, 062318 (2004).

[23] Y. Feng, R. Duan, and M. Ying, Phys. Rev. A 70, 012308 (2004).

[24] U. Herzog and J. A. Bergou, Phys. Rev. A 70, 022302 (2004).

[25] U. Herzog, Phys. Rev. A 75, 052309 (2007).

[26] X.-F. Zhou, Y.-S. Zhang, and G.-C. Guo, Phys. Rev. A 75, 052314 (2007).

[27] M. Kleinmann, H. Kampermann, and D. Bruss, Phys. Rev. A 81, 020304 (2010).

[28] L. Vandenberghe and S. Boyd, SIAM Rev. 38, 49 (1996).

[29] M. Yamashita, K. Fujisawa, and M. Kojima, Optim. Meth. Softw., 18, 491 (2003).

[30] T. Hashimoto, A. Hayashi, M. Hayashi, and M. Horibe, Phys. Rev. A 81, 062327 (2010). 